\title{
A novel and precise time domain description of MOSFET low frequency noise due to random telegraph signals
}

\author{
Roberto da Silva, ${ }^{1}$ Gilson Inacio Wirth, ${ }^{2}$ and Lucas Brusamarello ${ }^{1}$ \\ ${ }^{1}$ Instituto de Informatica \\ Universidade Federal do Rio Grande do Sul \\ Av Bento Gonçalves, 9500 \\ 91501-970 - Porto Alegre RS, Brasil \\ ${ }^{2}$ Escola de Engenharia \\ Universidade Federal do Rio Grande do Sul \\ Osvaldo Aranha, 103, 20 andar \\ 90035-190, Porto Alegre, RS, Brasil
}

\begin{abstract}
Nowadays, random telegraph signals play an important role in integrated circuit performance variability, leading for instance to failures in memory circuits. This problem is related to the successive captures and emissions of electrons at the many traps stochastically distributed at the silicon-oxide $\left(\mathrm{S}_{\mathrm{i}}-\mathrm{S}_{\mathrm{i}} \mathrm{O}_{2}\right)$ interface of MOS transistors. In this paper we propose a novel analytical and numerical approach to statistically describe the fluctuations of current due to random telegraph signal in time domain. Our results include two distinct situations: when the density of interface trap density is uniform in energy, and when it is an u-shape curve as prescribed in literature, here described as simple quadratic function. We establish formulas for relative error as function of the parameters related to capture and emission probabilities. For a complete analysis experimental u-shape curves are used and compared with the theoretical aproach.
\end{abstract}

Low frequency (LF) noise is a performance limiting factor for deep sub-micron CMOS devices [2]. In these devices, LF noise is dominated by multiple Random Telegraph Signals (RTS) (see [4] for a discussion on RTS in $\mathrm{S}_{\mathrm{i}}-\mathrm{S}_{\mathrm{i}} \mathrm{O}_{2}$ interfaces). The MOS transistor employed in analog and digital designs drives a current $I_{D S}$ which is a function of the potential differences $V_{D S}$ and $V_{G S}$ applied respectively between drain and source and gate and source[1]. RTS noise is due to succeeding electron capture and emission by a number of $N_{t r}$ traps distributed

Typeset by REVTEX 
according to a Poisson distribution at the $\mathrm{S}_{i}-\mathrm{S}_{i} \mathrm{O}_{2}$ interface, as it can be observed in plot 1 (a). This phenomena causes oscillations in the transistor current $I_{D S}$ over time, even with $V_{D S}$ and $V_{G S}$ constant. A typical variation in the current caused by one single trap is shown in figure 1 (b).
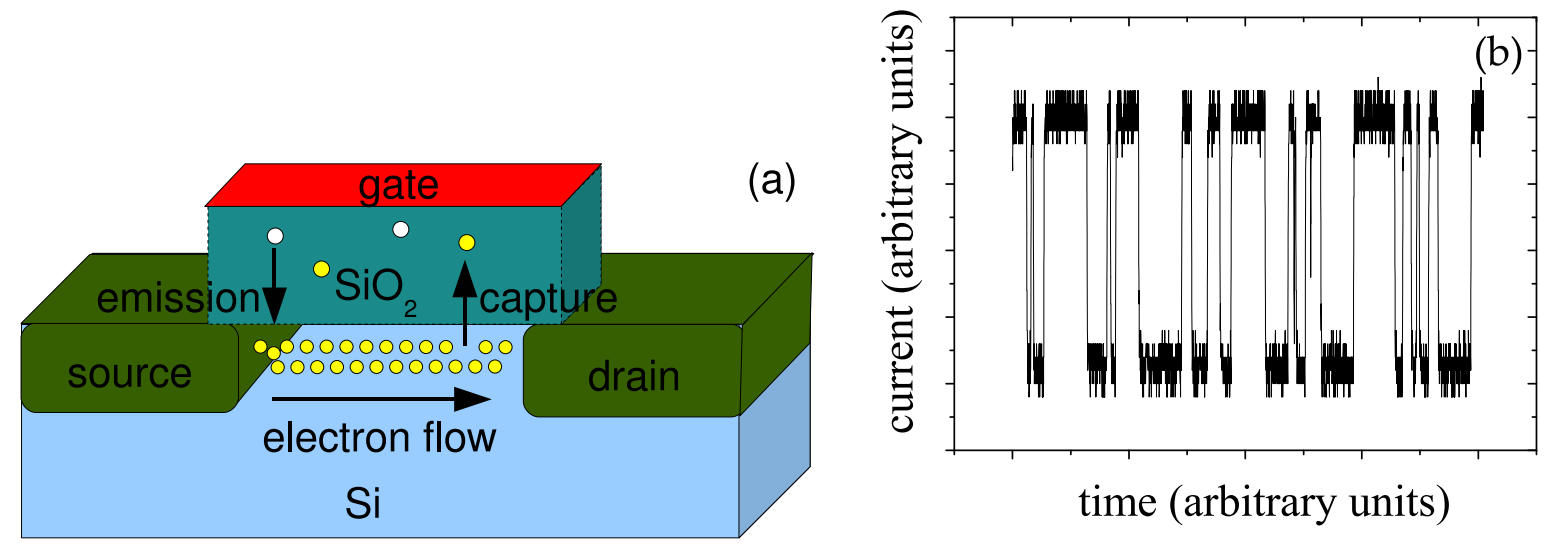

FIG. 1: Plot a: Physical mechanism for the generation of LF-noise; Plot b: This figure shows the effects caused by only one trap according to physical mechanism described in plot a. A discontinuity in the electrical current corresponds to the absorption or emission of an electron.

Noise performance may strongly vary between different devices on a same chip, and even between different operation points of a single transistor. Recent results [9] claim these effects responsible for fails in flash memories. For future technologies, RTS may become relevant also for the performance of combinational digital gates, as inverters and other logic gates. The propagation delay of a gate depends on the capability of its transistors to drive current. A smaller current driven by the transistor means larger propagation delay, which may lead to timing violations (failures). Hence, the variation in transistor drive current due to RTS may lead to circuit failures in future technology generations, and statistical modeling of random telegraph signal is demanded. But until now statistical models for RTS focused on frequency domain, for instance refer to [3] for a statistical RTS model in frequency domain. This is suitable for analog circuits, whose design and analysis is performed in frequency domain. However, for digital circuits an appropriate time domain statistical analysis is needed, since these circuits are analyzed and designed using time domain metrics. So, as RTS is becoming a major issue on upcoming digital integrated circuits, an approppriate RTS noise model for 
time domain is mandatory for the correct modeling of the behavior of fugure digital circuits.

Aiming to address this issue, this work presents for the first time in literature a comprehensive model for RTS in time domain. We derive analytical expressions for the average and relative error of the transistor current. We also analyze the joint contribution of all the traps to the transistor current, instead of analyzing only the current fluctuation due to one single trap, which is more suitable for analyzing the total current fluctuation of the transistor. Our model considers the density of states in the band-gap analyzing three possible situations:

1. The density of states is uniform in the band-gap range. This is based on previous results from literature (see for example [2])

2. The density of states curve measured from experimental data, which was published in [8] and shows that the density of states follows an u-shape curve. We performed a polynomial fit with a 8-degree polynomial which fits very well with the experimental data.

3. The density of states modeled as a quadratic function in the band-gap range. This is an approximation for the experimental data of [8], which is an u-shape curve.

In the random telegraph signals, $\sigma_{i}=0,1$ denotes the state of the i-th trap $(0=$ empty or 1 =occupied), the Fermi-Dirac statistics governs the probability of transition:

$$
\begin{aligned}
& \operatorname{Pr}\left(\sigma_{i}=0 \rightarrow \sigma_{i}=1\right) d t=\frac{d t}{10^{p_{i}}\left[1+\exp \left(-q_{i}\right)\right]}=\frac{d t}{\tau_{c}^{(i)}} \\
& \operatorname{Pr}\left(\sigma_{i}=1 \rightarrow \sigma_{i}=0\right) d t=\frac{d t}{10^{p_{i}}\left[1+\exp \left(q_{i}\right)\right]}=\frac{d t}{\tau_{e}^{(i)}}
\end{aligned}
$$

where $\tau_{c}^{(i)}$ and $\tau_{e}^{(i)}$ are respectively the average time of capture and emission and $q_{i}=$ $\left(E_{T}^{(i)}-\mu\right) / k_{B} T$ where $E_{T}^{(i)}$ is the energy ( within the band-gap) of the $i-$ th trap, $\mu$ is the fermilevel energy, $k_{B}=1.3806568 \times 10^{-23} \mathrm{Jk}_{B}^{-1}$ the Boltzmann constant and $T$ is temperature. In a first model $-Q<q_{i}<Q$ is considered a uniform random variable and $\tau_{c}^{(i)}, \tau_{e}^{(i)}$ are identically distributed, i.e., $\left\langle\tau_{c}^{(i)}\right\rangle=\left\langle\tau_{c}\right\rangle$ and $\left\langle\tau_{e}^{(i)}\right\rangle=\left\langle\tau_{e}\right\rangle$ for $i=1,2, \ldots, N_{t r}$.

Here, $p_{i}$ is also a random uniform variable within an interval $p_{\min }<p_{i}<p_{\max }$ and in this case in the frequency domain, we can establish an important connection. It is not difficult to show (see for example [4 -7]) that the power spectrum density correponding to the noise from the $i$-th trap is a Lorentzian function $S_{i}\left(f_{i}\right)=\left(A_{i}^{2} / f_{i}\right)\left[1+\left(f / f_{i}\right)^{2}\right]^{-1}$ where 
$f_{i}=1 / \tau_{c}^{(i)}+1 / \tau_{e}^{(i)}$ is the corner frequency corresponding to the trap and $A_{i}$ is its amplitude. From that we conclude $f_{i}=10^{-p_{i}}$ and due to this $f_{i}$ is uniformly distributed in a $\log _{10}$ scale resulting in a probability distribution $h\left(f_{i}\right)=\left[\ln 10\left(p_{\max }-p_{\min }\right) f_{i}\right]^{-1}$ for the corner frequencies (this assumption will be used from now on in this paper).

From this approach, we can calculate

$$
\operatorname{Pr}\left(\sigma_{i}=1\right)=\frac{1}{\tau_{e}} /\left(\frac{1}{\tau_{c}}+\frac{1}{\tau_{e}}\right)=\frac{\tau_{c}}{\tau_{c}+\tau_{e}}
$$

and thus the average current due to a single trap is computed by

$$
\begin{aligned}
\overline{I_{i}} & =\delta_{i} \cdot \operatorname{Pr}\left(\sigma_{i}=1\right)+0 \cdot \operatorname{Pr}\left(\sigma_{i}=0\right) \\
& =\frac{\tau_{c}^{(i)}}{\tau_{c}^{(i)}+\tau_{e}^{(i)}} \delta_{i}
\end{aligned}
$$

The amplitudes $\delta_{i}$ are also random variables and our results will be dependent on its first and second moments, respectively $\langle\delta\rangle$ and $\left\langle\delta^{2}\right\rangle$. The next step is to consider the contribution of all traps, because by doing so one can compute the fluctuation of the current passing through the channel of the MOS transistor. The average current, taking in consideration all random sources, is written as:

$$
\begin{aligned}
\langle\bar{I}\rangle=\left\langle\overline{I_{i}}\right\rangle & =\left\langle\sum_{i=1}^{N_{t r}} \overline{I_{i}}\right\rangle_{N_{t r}, \tau_{c}, \tau_{e}} \\
& =\frac{\langle\delta\rangle}{2 Q}\left(\sum_{N_{t r}=0}^{\infty} N_{t r} \frac{N^{N_{t r}} e^{-N}}{N_{t r} !}\right) \int_{-Q}^{Q} \frac{1+e^{-q}}{2(1+\cosh q)} d q \\
& =\frac{N\langle\delta\rangle}{2 Q}\left[2 Q+\ln \frac{\left(e^{-Q}+1\right)}{\left(e^{Q}+1\right)}\right] \\
& =\frac{N\langle\delta\rangle}{2 Q}\left[2 Q+\ln e^{-Q}\right]=\frac{N\langle\delta\rangle}{2}
\end{aligned}
$$

where $N=c\left(p_{\max }-p_{\min }\right)$ and $c=\ln 10 N_{\text {dec }} W L$, where $W$ and $L$ are the device dimensions and $\rho=\ln 10 N_{d e c}$ is the density of traps by area unit and by decade frequency in log-scale. So, we can conclude that $\langle\bar{I}\rangle$ is constant, i.e., it does not depend on $Q$. This conclusion motivates the investigation for superior moments of $\bar{I}$. 
Similarly we can compute the second moment:

$$
\begin{aligned}
\left\langle I^{2}\right\rangle= & \left\langle\left(\sum_{i=1}^{N_{t r}} \overline{I_{i}}\right)^{2}\right\rangle_{N_{t r}, \tau_{c}, \tau_{e}} \\
= & \left\langle\sum_{i=1}^{N_{t r}} \bar{I}_{i}^{2}+\sum_{i=1}^{N_{t r}} \sum_{\substack{j=1 \\
i \neq j}}^{N_{t r}} \overline{I_{i} I_{j}}\right\rangle_{N_{t r}, \tau_{c}, \tau_{e}} \\
= & \sum_{N_{t r}=0}^{\infty} \frac{e^{-N} N^{N_{t r}}}{N_{t r} !}\left(N_{t r}\left\langle\bar{I}^{2}\right\rangle_{\tau_{c}, \tau_{e}}\right. \\
& \left.+N_{t r}\left(N_{t r}-1\right)\langle\bar{I}\rangle_{\tau_{c}, \tau_{e}}^{2}\right)
\end{aligned}
$$

where $\left\langle\bar{I}_{i} \bar{I}_{j}\right\rangle_{\tau_{c}, \tau_{e}}=\left\langle\bar{I}_{i}\right\rangle_{\tau_{c}, \tau_{e}}\left\langle\bar{I}_{j}\right\rangle_{\tau_{c}, \tau_{e}}=\langle\bar{I}\rangle_{\tau_{c}, \tau_{e}}^{2}$.

So, this amount can be calculated:

$$
\begin{aligned}
\left\langle\bar{I}^{2}\right\rangle_{\tau_{c}, \tau_{e}} & =\frac{\delta^{2}}{2 Q} \int_{-Q}^{Q} \frac{\left(1+e^{-q}\right)^{2}}{4(1+\cosh q)^{2}} d q \\
& =\frac{\delta^{2}}{(2 Q)\left(e^{Q}+1\right)}\left(Q-e^{Q}+Q e^{Q}+1\right) \\
& =\delta^{2} \frac{Q-\tanh (Q / 2)}{2 Q} .
\end{aligned}
$$

Because $\sum_{N_{t r}=0}^{\infty} N^{N_{t r}} N_{t r} \frac{e^{-N}}{N_{t r} !}=N$ and $\sum_{N_{t r}=0}^{\infty} N_{t r}\left(N_{t r}-1\right) \frac{e^{-N}}{N_{t r} !}=N^{2}$, thus we find that

$$
\left\langle I^{2}\right\rangle=\frac{N\left\langle\delta^{2}\right\rangle}{2 Q}[Q-\tanh (Q / 2)]+\langle I\rangle^{2}
$$

what gives $\operatorname{var}(I)=\left\langle I^{2}\right\rangle-\langle I\rangle^{2}=\frac{N\left\langle\delta^{2}\right\rangle}{2 Q}[Q-\tanh (Q / 2)]$. And thus the relative error is described by

$$
\begin{aligned}
e(I) & =\frac{\sqrt{v a r(I)}}{\langle I\rangle} \\
& =\left(2 \frac{\left\langle\delta^{2}\right\rangle}{\langle\delta\rangle^{2}}\right)^{1 / 2} N^{-1 / 2}\left[\frac{Q-\tanh (Q / 2)}{Q}\right]^{1 / 2} \\
& =\left(2 \frac{\left\langle\delta^{2}\right\rangle}{\langle\delta\rangle^{2}}\right)^{1 / 2} \frac{\left(\ln 10 N_{\text {dec }} W L\right)^{-1 / 2}}{\left(p_{\max }-p_{\min }\right)^{1 / 2}}\left[\frac{Q-\tanh (Q / 2)}{Q}\right]^{1 / 2} \\
& =K\left(\frac{Q-\tanh (Q / 2)}{Q}\right)^{1 / 2}
\end{aligned}
$$


where $K=\left(2 \frac{\left\langle\delta^{2}\right\rangle}{\langle\delta\rangle^{2}}\right)^{1 / 2} \frac{\left(\ln 10 N_{d e c} W L\right)^{-1 / 2}}{\left(p_{\max }-p_{\min }\right)^{1 / 2}}$.

Thus by considering the variable $q=\left(E_{T}-\mu\right) / k_{B} T$ being uniformly distributed, the relative error behaves as a simple logistic function of the band gap amplitude $(Q)$.

Aiming to propose a more realistic model then the supposition of the density of states following an uniform distribution, we propose an alternative model that considers the density of states as being quadratically distributed. The theoretical quadratic function is a reasonable approximation to experimental results [8] and is simpler than the polynomial fit to those experimental data. For this, the theoretical $u$-shape modeled as a quadratic function defined in the interval $[-Q, Q]$ is here proposed:

$$
\operatorname{Pr}(q)=a q^{2}+c
$$

Supposing normalization $\int_{-Q}^{Q} \operatorname{Pr}(q) d q=1$, i.e., $2 a Q^{3} / 3+2 c Q=1$, we have

$$
\operatorname{Pr}(q)=a q^{2}+\frac{\left(1-2 a Q^{3} / 3\right)}{2 Q}
$$

and from the existence condition $c=\left(1-2 a Q^{3} / 3\right)>0$ and then $a<3 /\left(2 Q^{3}\right)$

Following the same procedure used before (density of states uniformly-distributed) now applied to the case which the density of states follows a quadratic curve, we first calculate the first moment and then compute the average of current considering the random telegraph noise caused by all traps in the interface:

$$
\begin{aligned}
\langle I\rangle= & \langle\delta\rangle N \int_{-Q}^{Q} \frac{1+e^{-q}}{2(1+\cosh q)} \operatorname{Pr}(q) d q \\
& \frac{\langle\delta\rangle N}{2} \int_{-Q}^{Q} \frac{\left(1+e^{-q}\right)\left[a q^{2}+\frac{\left(1-2 a Q^{3} / 3\right)}{2 Q}\right]}{(1+\cosh q)} d q
\end{aligned}
$$

This result must be obtained by using numerical methods for each $a$. For $a \rightarrow 0$, we have the expected $\langle I\rangle \rightarrow \frac{N\langle\delta\rangle}{2}$. We tested many values $a<3 /\left(2 Q^{3}\right)$ and no meaningful deviates of $\langle I\rangle=\frac{N\langle\delta\rangle}{2}$ were observed. Indeed just corrections of order $O\left(10^{-7}\right)$ were verified, and there are no evidences to believe there might be large deviations for the current considering our theoretical $\mathrm{u}$-shape curve. Our tests were performed using $Q_{\max }=10$, and to perform it, just values $a<3 /\left(2 \cdot 10^{3}\right)=0.0015$ were considered. We have employed the Simpsom rule (see for example [10]) for the numeric integration of equation 3. The reader might think $a$ 
is very small and differences would be observed at higher values of $a$. In order to clarify this issue, we experimented $a=0.01$ up to $a=0.1874<3 /\left(2 \cdot 2^{3}\right)=0.1875$ corresponding to $Q_{\max }=2$ and the same conclusion was obtained: $\langle I\rangle$ is constant under $Q$ variations. Thus, the relationship between $\langle I\rangle$ and $Q$ is independent of $a$.

The most accurate model comes from the actual density of states, which can be measured from experimental data as in [8] and fitted by a polynomial. We have verified the differences in the MOSFET current fluctuations between modeling the band-gap as a uniform distribution, a theoretical $\mathrm{u}$-shape modeled by the simple quadratic function described by equation 2 and the actual experimental data fitted by a $8^{\text {th }}$-degree polynomial.

The procedure of using the experimental density of states consists of performing a scan of the figures of the experimental density of states found in [8], i.e., the density of traps having energy $E_{T}$, corresponding to $q=\left(E_{T}-\mu\right) / k_{B} T$, here denoted by $f(q)$.

In that work $3 \mathrm{u}$-shape curves are studied, $f_{1}(q) \times q, f_{2}(q) \times q$ and, $f_{3}(q) \times q$, corresponding to 3 different oxides (in the nomenclature of ref. [8], reoxidized: oxide I, nitrided: oxide II and, tceoxide: oxide III) defined in a symmetric band gap interval $[-\alpha, \alpha]$. The first step was to map this band gap to an interval $[-Q, Q]$, what was done with the simple transformation $q^{\prime}=Q\left[1-\frac{(\alpha-q)}{\alpha}\right]$. For our scanning $n=100$ points, $\left\{\left(q_{i}, f\left(q_{i}\right)\right)\right\}_{i=1}^{n}$, were collected from experimental figures and fitted by a 8-degree polynomial $\left(g_{i}\left(q^{\prime}\right)=\sum_{k=0}^{8} \beta_{k} q^{\prime}{ }^{k}\right)$. The degree of 8 was used because it presents a good visual fit for all u-shape curves. A normalization is required, and thus $\operatorname{Pr}_{i}\left(q^{\prime}\right)=g_{i}\left(q^{\prime}\right) / \int_{-Q}^{Q} g_{i}\left(q^{\prime}\right) d q^{\prime}=\sum_{k=1}^{8} \beta_{k}^{\prime} q^{\prime k}$, where $\beta_{k}^{\prime}=\beta_{k} /\left(2 \sum_{j=0}^{4} \beta_{2 j+1}(2 j+1)^{-1} Q^{2 j+1}\right)$, for $q^{\prime} \in[-Q, Q]$. And so a polynomial fit was performed for each value attributed to $Q$ changing from $Q_{\min }$ to $Q_{\max }$ and after we have the experimental probability distribution describing the density of states. Next we perform numerical integrations of the equations for the current and the relative error obtained in this manuscript, which are performed using Simpsom method with a very small step $\left(h \sim 10^{-3}\right)$. It supplies high precision for the integration and so for our estimates. These simple procedures are implemented in Fortran.

Figure 2 shows the experimental u-shapes. The inside figure corresponds to an example of good polynomial fit performed for the one of the oxides (tceoxide). So we perform calculations to obtain $\langle I\rangle$.

In figure 3 we can observe that $\langle I\rangle$ decays as function of $Q$ which is not observed when the density of states is uniform or even when the curve is the theoretical u-shape. This 


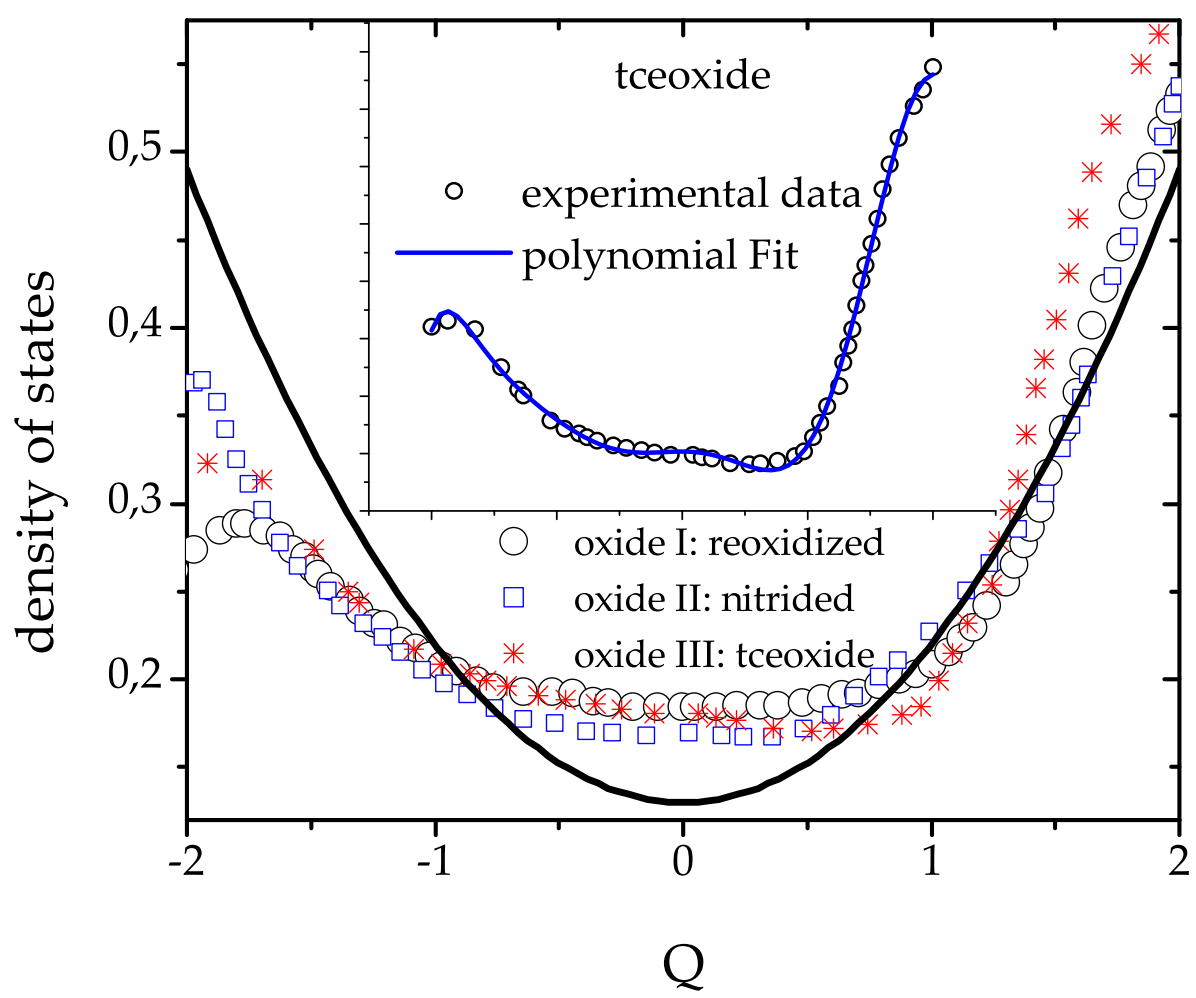

FIG. 2: Experimental density of states (U-shape curves) for 3 different oxides. The data was extracted from ref. [8]. The inside plot corresponds to a example of the good polynomial fit by a nine degree polynomial for the oxide III (tceoxide in the nomenclature of [8]). Just for a comparisom the continuous curve represents a theoretical curve for $a=0.09$ for the same $Q=2$.

corroborates the idea that the asymmetry of the u-shape might be important to observe the current differences that appear when $Q$ changes. However, it is important to notice that the average noise current decays at most 10\% (for the tceoxide), for high values of Q. Since Q for $\mathrm{S}_{i} \mathrm{O}_{2}$ is approximately 2 , in this case the decrease is nearly $5 \%$. It is also important to analyze the relative error in the current by computing its higher moments.

We then calculate the second moment for the theoretical u-shape density:

$$
\left\langle\bar{I}^{2}\right\rangle_{\tau_{c}, \tau_{e}}=\left\langle\delta^{2}\right\rangle \int_{-Q}^{Q} \frac{\left(1+e^{-q}\right)^{2}\left[a q^{2}+\frac{\left(1-2 a Q^{3} / 3\right)}{2 Q}\right]}{4(1+\cosh q)^{2}} d q
$$




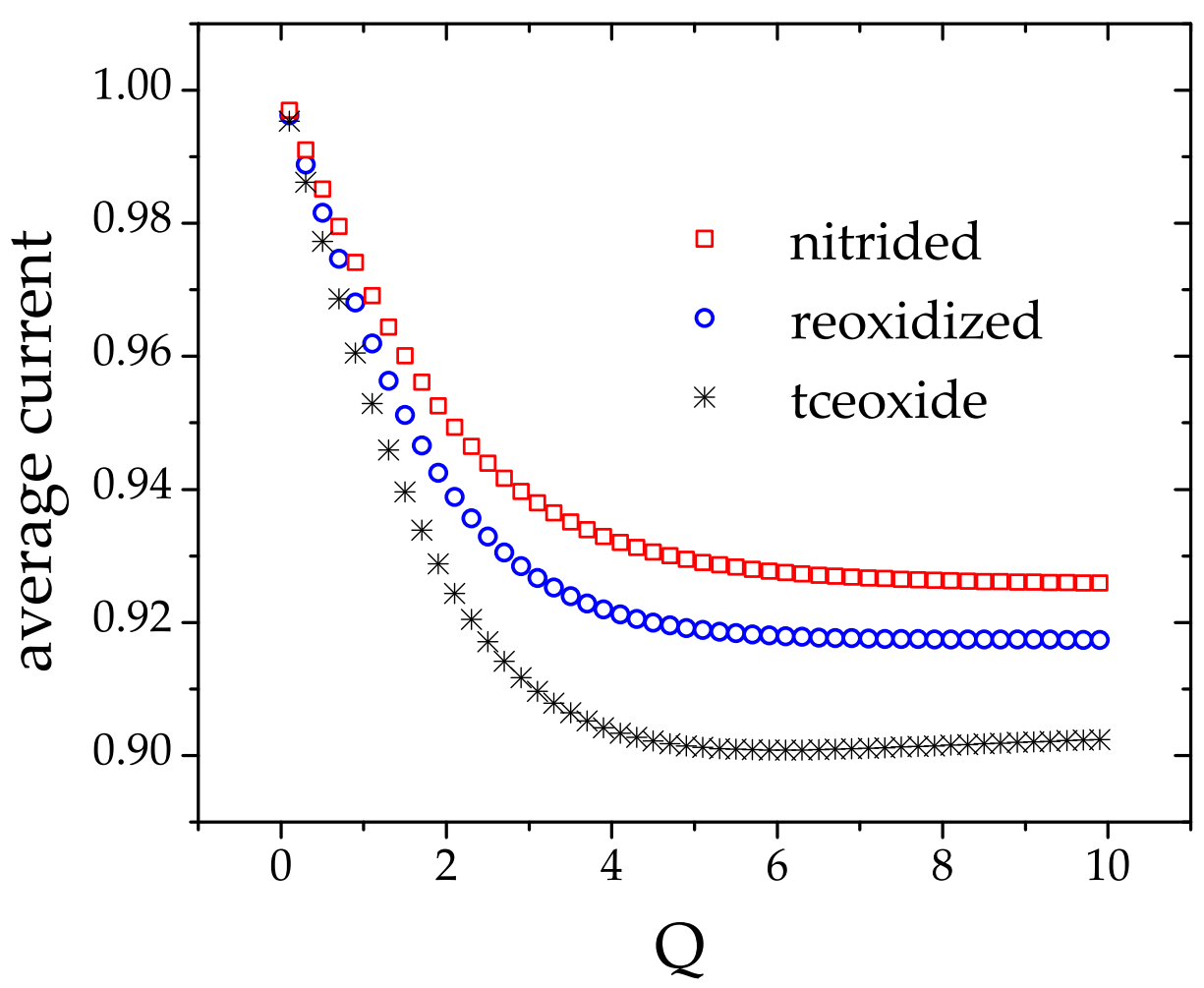

FIG. 3: Average current using the 3 experimental density of states shown in figure 2 as function of $Q$. The results show a decay which is not observed when the density of states is uniform or even when our theoretical u-shape density of states is considered.

and the variance is

$$
\operatorname{var}(I)=N\left\langle\delta^{2}\right\rangle \int_{-Q}^{Q} \frac{\left(1+e^{-q}\right)^{2}\left[a q^{2}+\frac{\left(1-2 a Q^{3} / 3\right)}{2 Q}\right]}{4(1+\cosh q)^{2}} d q .
$$

We can calculate also in this case the relative error after a few algebraic manipulation we have

$$
e(I)=K \frac{\left(\int_{-Q}^{Q} \frac{\left(1+e^{-q}\right)^{2}\left[a q^{2}+\frac{\left(1-2 a Q^{3} / 3\right)}{2 Q}\right]}{(1+\cosh q)^{2}} d q\right)^{1 / 2}}{\int_{-Q}^{Q} \frac{\left(1+e^{-q}\right)\left[a q^{2}+\frac{\left(1-2 a Q^{3} / 3\right)}{2 Q}\right]}{(1+\cosh q)} d q}
$$

where $K=\left(2 \frac{\left\langle\delta^{2}\right\rangle}{\langle\delta\rangle^{2}}\right)^{1 / 2} \frac{\left(\ln 10 N_{d e c} W L\right)^{-1 / 2}}{\left(p_{\max }-p_{\min }\right)^{1 / 2}}$. Integrating numerically the last equation we have 
in the left plot of figure 4 the behavior of $e(I)$ as function of $Q$, for different value of $a<0.0015$ showing that the relative error is sensitive for different and small (possible) values of $a$. For a suitable comparison, it is plotted in the same figure the case $a=0$, which corresponds to the uniform distribution of density of states. The inside plot shows the same plot in a log-log scale. For higher values of $a$ a power law behavior can be adopted to describe the $e(I) \times Q$. The left plot of the same figure 4 describes that the power law fit $e(I)=a Q^{\theta}$ considering $Q>2$ is suitable simple fit for this interval. We find the best estimate $\theta=0.143(1)$ and $a=1.013(1)$.
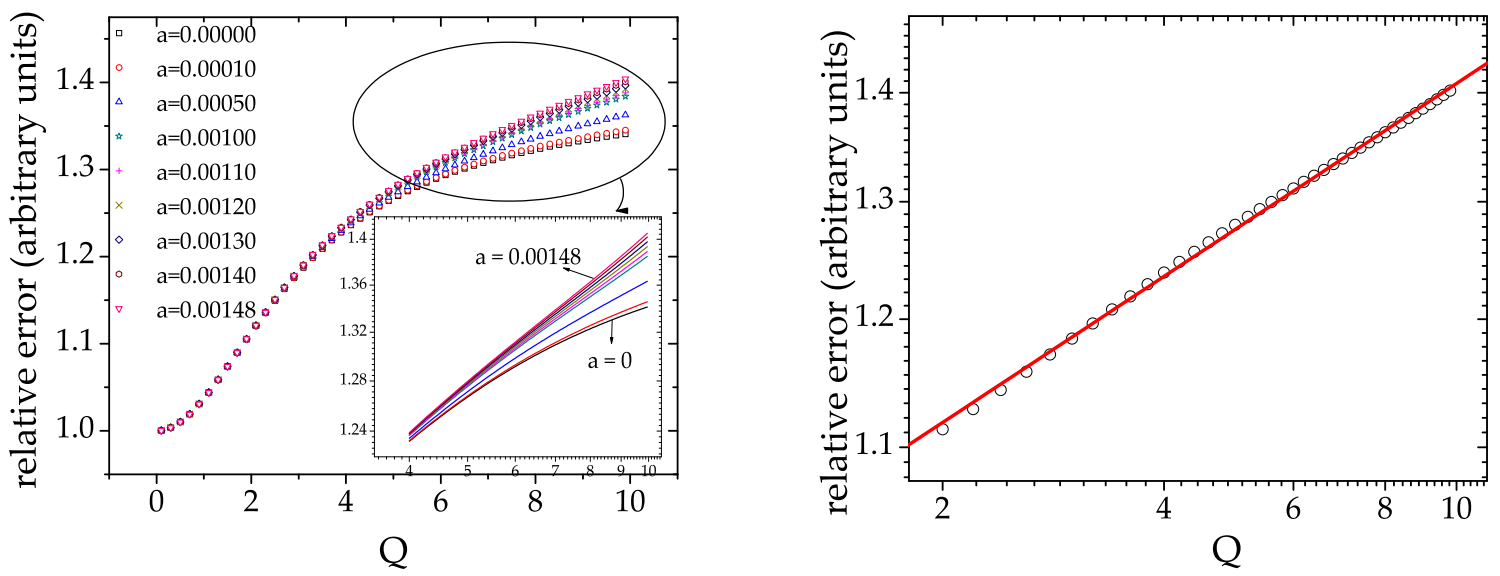

FIG. 4: Left plot: Relative error as function of $Q$ for a u-shape(quadratic) density of states. The inside plot shows a log-log plot for $Q>4$ showing that higher values of $a$ leads to a power law behavior of the relative error as function of $Q$. Right plot A power law fit of relative error as funcion of $Q$. It was used $a=0.00148$ and $Q>2$ for the plots.

These results show that the relative error in the current is not universal since the relative error shows different behavior depending on the density of states. Finally we have performed the same calculations for the relative error considering the experimental u-shapes, as shown in figure 5. We observe a difference in the dependence of $Q$ between the 3 different ushapes. A similar behavior (logistic curve), predicted for the uniform case (see equation 1), is observed using the experimental u-shapes, however there is a sensitive difference for higher values of $Q$ between the two cases. Our theoretical approach for the u-shape supplies exactly an intermediate behavior between the uniform and experimental u-shape results, 
showing it is suitable for modeling RTS noise in MOSFETs because it is simpler then the higher-order polynomial fit and more accurate than the uniform case!

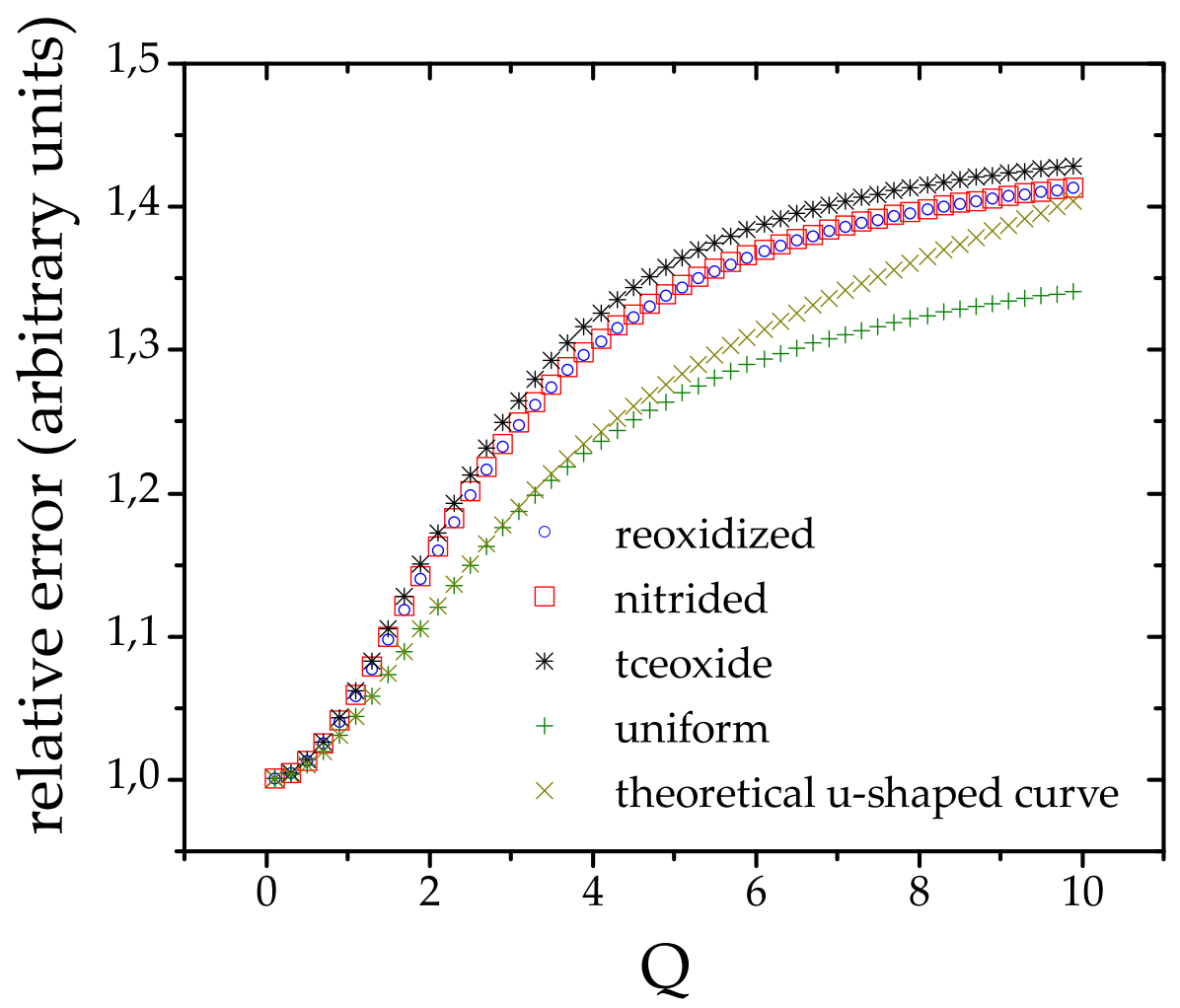

FIG. 5: Relative error as function of $Q$ for the experimental u-shapes.

Summarizing, we developed an original methodology in time domain to model the noise behavior due to traps at the $\mathrm{S}_{i}-\mathrm{S}_{i} \mathrm{O}_{2}$ interface. Our results show that the relative error depends on the amplitude $(Q)$ of the density of states at the interface.

Our model for the average current and its relative error depends upon the model of the density of states adopted. When considering the density of states as having uniform or quadratic density of trap energies in the band gap we conclude that the relative error behaves as a logistic function in relation to Q. For the density of states modeled as an uniform distribution, a very simple formulation can still capture a logistic behavior similar to the experimental data.

The experimental $\mathrm{u}$-shapes show a decay in the average current not observed when the theoretical $u$-shape is considered. Such results represent an important advance in modelling RTS at device and circuit levels, and experiments can be performed to check these behaviors. 
We believe that device designers can use the models proposed in this work for modeling the relative error as a function of the band-gap in upcoming technologies.

\section{Acknowledgments}

We would like to thank the anonymous referees for the helpful suggestions. R. da Silva and G. I. Wirth are financially supported by the following projects of CNPq: 490440/2007, 480258/2008-2, 311343/2006-6, and 577473/2008-5.

[1] B.J. Sheu, D.L. Scharfetter, P.K. Ko, M.C.Jeng, BSIM: Berkeley short-channel IGFET model for MOS transistors, IEEE Journal of Solid-State Circuits, 22, 558 (1987),

[2] M.J. Kirton, M.J. Uren, Noise in solid-state microstrutures: A new perspective on individual defects, interface states and low-frequency (1/f) noise, Advances in Physics 38, 4, 367-468 (1989)

[3] R.da Silva, G.I.Wirth, L.Brusamarello, An appropriate model for the noise power spectrum produced by traps at the $\mathrm{Si}-\mathrm{SiO} 2$ interface: a study of the influence of a time-dependent Fermi level, Journal of Statistical Mechanics: Theory and Experiment, P10015/1-P10015/10 (2008).

[4] Machlup, "Noise in Semiconductors: Spectrum of a Two-Parameter Random Signal", J. Applied Physics 35, 3, 341-343 (1954)

[5] G. Wirth, R.da Silva, J. Koh, R. Thewes, R. Brederlow, Modeling of Statistical low-frequency noise of deep-submicrometer MOSFETs, IEEE Trans. Electron. Devices 52, 7 1576-1588 (2005)

[6] R. da Silva, G. I. Wirth, R. Brederlow, Novel analytical and numerical approach to modeling low-frequency noise in semiconductor devices, Physica A 362, 277-288 (2006)

[7] G Wirth, R da Silva and R Brederlow, "Statistical Model for the Circuit Bandwidth Dependence of Low-Frequency Noise in Deep-Submicrometer MOSFETs"; IEEE Trans. Electron. Devices 54, 340-345 (2007)

[8] H. Wong, Y. C. Cheng, Study of the eletronic trap distribution at the $\mathrm{SiO}_{2}-\mathrm{S}_{\mathrm{i}}$ interface utilizing the low-frequency noise measurement, IEEE Transactions on electron devices 37, 7, 1743-1749 (1990) 
[9] C. M. Compagnoni, R. Gusmeroli, A. S. Spinelli, A. L. Lacaita, M. Bonanomi, A. Visconti, Statistical Model for Random Telegraph Noise in Flash Memories, IEEE Transactions on electron devices 55, 1, 388-395 (2008)

[10] W. Press et al., Numerical Recipes (Cambridge University Press, London, 1986). 